Commun. math. Phys. 24, 253-259 (1972)

(C) by Springer-Verlag 1972

\title{
On the Spontaneous Magnetization in the Ising Model
}

\author{
ANDERS MARTIN-LÖF \\ Rockefeller University, Department of Mathematics, New York, N.Y., USA
}

Received October 25, 1971

\begin{abstract}
The equality between the spontaneous magnetization and the "long range order" is established for the Ising model with nearest neighbour interactions for low and high temperatures. The proof is presented for the two-dimensional lattice but works also in higher dimensions. The result verifies that the value $m^{*}=\left(1-(\operatorname{Sh} \beta)^{-4}\right)^{1 / 8}$ of the spontaneous magnetization below the critical point calculated by Onsager and Yang is the true value, which has been a long standing open question.
\end{abstract}

\section{Introduction}

We consider an Ising spin system in a finite rectangular box $\Lambda$ on the two-dimensional lattice $Z^{2}$, which contains $N \times M$ points, $N \leqq M$, and is centered at the origin. I.e. at each point $p \in \Lambda$ there is a spin $\sigma_{p}$ taking values \pm 1 , and the energy of a spin configuration is given by:

$$
-E=J \sum_{p, q \in \Lambda}^{\prime} \sigma_{p} \sigma_{q}+H \sum_{p \in \Lambda} \sigma_{p}+\text { boundary term }
$$

where $J>0$ is the strength of the interaction, $H$ the external magnetic field and $\sum_{p, q \in \Lambda}^{\prime}$ denotes the sum over all pairs of nearest neighbours in $\Lambda$. We are going to consider four different boundary conditions: $\Lambda$ being surrounded by fixed spins all having the value +1 or $-1, \Lambda$ being a torus, i.e. the spins at opposite edges of $\Lambda$ interact as if they were nearest neighbours, or free boundary spins, i.e. there are no spins outside $\Lambda$. These four cases will be denoted by,,$+- t$ or $f$. The boundary term in (1) consists of the energy of interaction with the fixed spins or between the opposite spins in the edges respectively. The probability of a spin configuration in $\Lambda$ is then proportional to the Bolzmannfactor $e^{-\frac{E}{k T}}$. We put $\frac{2 J}{k T}=\beta$ and $\frac{H}{k T}=h$ and denote the spin correlations $\left\langle\prod_{p \in A} \sigma_{p}\right\rangle$ by $\left\langle\sigma_{A}\right\rangle_{h, A, b}$ for $A \subseteq \Lambda$ and $b=+,-, t$ or $f$. We will also consider the correlations for an infinite system and denote them by:

$$
\left\langle\sigma_{A}\right\rangle_{h, b}=\lim _{N \rightarrow \infty}\left\langle\sigma_{A}\right\rangle_{h, A, b} \text { for } A \text { finite } \subset Z^{2} .
$$


When $h=0$ we will sometimes drop it in the notation:

$$
\left\langle\sigma_{A}\right\rangle_{0, \Lambda, b}=\left\langle\sigma_{A}\right\rangle_{A, b} .
$$

It will be very useful in the following to represent a configuration in $\Lambda$ by specifying the boundaries between the regions with + spins and with - spins. This can be done as follows: At the midpoint of each line segment joining a pair of opposite spins we draw a perpendicular segment of length 1 centered there and obtain a family of lines on the dual lattice, which can be drawn as $Z^{2}$ translated by the amount $(1 / 2,1 / 2)$. The lines separate the + spins and the - spins, and with the three boundary conditions,+- or $t$ they form a closed graph, i.e. only an even number of lines can meet at each point of the dual lattice. The graph is contained in the box of size $N \times M$ corresponding to $\Lambda$ on the dual lattice if the boundary condition is,+- or $f$ and in the toroidal box of size $N \times M$ corresponding to $\Lambda$ if the boundary condition is $t$. This corresponding box we denote by $\bar{\Lambda}$. With the boundary condition $f$ only an even number of lines can meet at interior points of $\bar{\Lambda}$, each boundary point can only be contained in 0 or 1 line, and no line can connect two boundary points. If we furthermore modify the lines at points where four of them meet as follows:

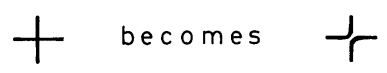

Fig. 1. Rule for the splitting into contours

we realize that with boundary condition,+- or $t$ the lines split into a family of closed disjoint edge-selfavoiding contours $\gamma_{1}, \ldots, \gamma_{n}$, which are allowed to touch each other and themselves as in Fig. 1 but not in any other way. When the boundary condition is $t$ there must be an even number of contours going around the torus. With boundary condition $f$ contours can also start and end at boundary points of $\bar{\Lambda}$. The correspondence between the family of contours and the configuration is $1-1$ when the boundary condition is + or - and $1-2$ when it is $t$ or $f$, and if $h=0$ the probability of a contour configuration $X=\left(\gamma_{1}, \ldots, \gamma_{n}\right)$ is proportional to $e^{-\beta \sum_{1}^{n}\left|\gamma_{i}\right|}$ if $X$ is allowed, where $|\gamma|$ denotes the length of the contour $\gamma$. When $h=0$ the system can thus be thought of as a "gas" of contours interacting via the restrictions mentioned above, each contour $\gamma$ having an "activity" $e^{-\beta|\gamma|}$.

The spontaneous magnetization, $m^{*}$, is the average magnetization of an infinite system in a vanishing field: $m^{*}=\lim _{h \rightarrow 0+} \lim _{N \rightarrow \infty}\left\langle\sigma_{p}\right\rangle_{h, \Lambda, b} .\left\langle\sigma_{p}\right\rangle_{h, b}$ $=\lim _{N \rightarrow \infty}\left\langle\sigma_{p}\right\rangle_{h, \Lambda, b}$ exists and does not depend on $b$ and $p$ if $h>0$ because 
there is a unique translation invariant equilibrium state when $h>0$ [10]. The long range order, $l_{b}^{*}$, is defined as $l_{b}^{*}=\lim _{|p-q| \rightarrow \infty} \lim _{N \rightarrow \infty}\left\langle\sigma_{p} \sigma_{q}\right\rangle_{0, \Lambda, b}^{1 / 2}$.

As we will see $\left\langle\sigma_{p} \sigma_{q}\right\rangle_{0, b}=\lim _{N \rightarrow \infty}\left\langle\sigma_{p} \sigma_{q}\right\rangle_{0, A, b}$ exists and is independent of $b$ and is translation invariant for low or high enough temperature, i.e. for large or small enough $\beta$. For the two-dimensional Ising model with boundary condition $t l_{t}^{*}$ has been calculated for all $\beta$ and has the value mentioned above for $\beta>\beta_{c}$ and is zero for $\beta \leqq \beta_{c}, \beta_{c}$ being determined by the equation $\operatorname{Sh} \beta_{c}=1$ or $e^{-\beta_{c}}=\sqrt{2}-1$.

\section{Main Result}

Our main result can now be formulated as follows:

Theorem 1. If $e^{-\beta}<1 / 3$ then $l_{b}^{*}$ does not depend on the boundary condition $b=+,-$, tor $f$ and is equal to $m^{*}$. Moreover the mean magnetization $m_{\Lambda}=\left|\frac{1}{|\Lambda|} \sum_{p \in \Lambda} \sigma_{p}\right|$ converges to $m^{*}$ in probability if $M \leqq N^{\alpha}$ for some $\alpha \geqq 1$.

Remark. It has been shown by Griffiths [6] and will also be seen below that $0 \leqq l_{f}^{*} \leqq l_{t}^{*} \leqq l_{+}^{*}=l_{-}^{*}=m^{*}$, so that $l_{b}^{*}=m^{*}=0$ if $m^{*}=0$. The estimates of Fisher [1] show that this happens if $\operatorname{th} \beta / 2<1 / 3$, so we know that $l_{b}^{*}=m^{*}$ outside the interval $1 / 3 \leqq e^{-\beta} \leqq 1 / 2$.

The proof of Theorem 1 will follow by combining the results of Lemma 1 and Theorem 2:

Lemma 1. $\left\langle\sigma_{A}\right\rangle_{+}=\lim _{N \rightarrow \infty}\left\langle\sigma_{A}\right\rangle_{0, \Lambda,+}$ exists for any finite $A$, and $\left\langle\sigma_{A}\right\rangle_{+}$ $=\lim _{h \rightarrow 0+}\left\langle\sigma_{A}\right\rangle_{h, b}$ for any boundary condition $b .\left\langle\sigma_{A}\right\rangle_{+}$is translation invariant and clustering, i.e. $\lim _{|a| \rightarrow \infty}\left\langle\sigma_{A+a} \sigma_{B}\right\rangle_{+}=\left\langle\sigma_{A}\right\rangle_{+}\left\langle\sigma_{B}\right\rangle_{+}$for any finite $A$ and $B$. In particular $m^{*}=\left\langle\sigma_{p}\right\rangle_{+}$for any $p$, and $l_{+}^{*}=m^{*}$.

Proof. The following elegant proof is due to (at least) Griffiths (private communication). It several times makes use of the Griffiths, Kelley, Sherman (G.K.S.) inequalities $[3,4,7,8]$, which imply that in the presence of an arbitrary inhomogenous non negative external field the correlations $\left\langle\sigma_{A}\right\rangle_{A}$ in any finite box $\Lambda$ are increasing functions of the fields at the different points and of the interaction strengths. This implies that

$$
\left\langle\sigma_{A}\right\rangle_{h, \Lambda^{\prime},+} \leqq\left\langle\sigma_{A}\right\rangle_{h, \Lambda,+} \quad \text { if } h \geqq 0 \text { and } \Lambda^{\prime} \supset \Lambda
$$

because the latter quantity can be obtained from the former by applying an infinite positive field at all points in $\Lambda^{\prime} \backslash \Lambda$. Thus $\left\langle\sigma_{A}\right\rangle_{h,+}=\lim _{N \rightarrow \infty}\left\langle\sigma_{A}\right\rangle_{h, \Lambda,+}$ exists, and $\left\langle\sigma_{A}\right\rangle_{h,+} \leqq\left\langle\sigma_{A}\right\rangle_{h, \Lambda,+}$ for $h \geqq 0$. Furthermore $\left\langle\sigma_{A}\right\rangle_{h,+}$ is in$19^{*}$ 
creasing in $h$ for $h \geqq 0$. If we let $h \rightarrow 0+$ and then $N \rightarrow \infty$ we get from the last inequality: $\lim _{h \rightarrow 0+}\left\langle\sigma_{A}\right\rangle_{h+} \leqq\left\langle\sigma_{A}\right\rangle_{+}$. The monotonicity in $h$ on the other hand gives $\lim _{h \rightarrow 0+}\left\langle\sigma_{A}\right\rangle_{h,+} \geqq\left\langle\sigma_{A}\right\rangle_{+}$so equality is established. From [10] we know that there is only one translation invariant equilibrium state when $h>0$, which fact implies that $\left\langle\sigma_{A}\right\rangle_{h,+}=\left\langle\sigma_{A}\right\rangle_{h, b}$ for any boundary condition $b$, and that it is translation invariant. $\left\langle\sigma_{A}\right\rangle_{+}$is then also translation invariant.

To prove the clustering property we note that by G.K.S.

$$
\left\langle\sigma_{A+a} \sigma_{B}\right\rangle_{+} \geqq\left\langle\sigma_{A}\right\rangle_{+}\left\langle\sigma_{B}\right\rangle_{+} .
$$

If $|a|$ is large enough we can however enclose $A+a$ and $B$ in two disjoint boxes $\Lambda_{1}+a$ and $\Lambda_{2}$. If we impose + boundary conditions in these $\left\langle\sigma_{A+a} \sigma_{B}\right\rangle$ can only increase by G.K.S. With these boundary conditions the boxes become independent however, so we get:

$$
\left\langle\sigma_{A+a} \sigma_{B}\right\rangle_{+} \leqq\left\langle\sigma_{A+a}\right\rangle_{A_{1}+a,+}\left\langle\sigma_{B}\right\rangle_{A_{2},+}=\left\langle\sigma_{A}\right\rangle_{A_{1},+}\left\langle\sigma_{B}\right\rangle_{A_{2},+},
$$

and

$$
\varlimsup_{|a| \rightarrow \infty}\left\langle\sigma_{A+a} \sigma_{B}\right\rangle_{+} \leqq\left\langle\sigma_{A}\right\rangle_{A_{1},+}\left\langle\sigma_{B}\right\rangle_{A_{2},+}
$$

But $\Lambda_{1}$ and $\Lambda_{2}$ can be chosen arbitrarily large, so we get

$$
\lim _{|a| \rightarrow \infty}\left\langle\sigma_{A+a} \sigma_{B}\right\rangle_{+}=\left\langle\sigma_{A}\right\rangle_{+}\left\langle\sigma_{B}\right\rangle_{+} .
$$

Remark. The G.K.S. inequalities also imply that

$$
\left\langle\sigma_{A}\right\rangle_{f} \leqq\left\langle\sigma_{A}\right\rangle_{t} \leqq\left\langle\sigma_{A}\right\rangle_{+}=\left\langle\sigma_{A}\right\rangle_{-}
$$

for $|A|$ even, which proves the inequality of the remark after Theorem 1.

Theorem 2. If $e^{-\beta}<1 / 3$ then $\left\langle\sigma_{A}\right\rangle_{b}$ is independent of $b=+,-, t$ or $f$ if $|A|$ is even. In particular $\left\langle\sigma_{p} \sigma_{q}\right\rangle_{b}$ is independent of $b$, and thus also $l_{b}^{*}$.

Proof. Because of the previous remark we need only prove that $\left\langle\sigma_{A}\right\rangle_{f} \geqq\left\langle\sigma_{A}\right\rangle_{+}$if $|A|$ is even. We now use the representation of the spin system as an ensemble of contours in the box $\bar{\Lambda}$ on the dual lattice. $A$ is going to be far from $\partial \Lambda$ when $N$ is large; we can assume that $d(A, \partial \Lambda) \geqq N^{1 / 2}$ e.g. We next give an estimate of the boundary effects with boundary condition $f$ which will be proved after Theorem 2:

Lemma 2. Let $E$ be the event defined by the condition: No contour with endpoints on $\partial \bar{\Lambda}$ separates any two points in $A$. Then $P_{\Lambda, f}(E) \rightarrow 1$ uniformly when $d(A, \partial \Lambda) \geqq N^{1 / 2}$ as $N \rightarrow \infty$ if $e^{-\beta}<1 / 3$.

Because $\left\langle\sigma_{A}\right\rangle_{A, f}=P_{A, f}(E)\left\langle\sigma_{A}\right\rangle_{\Lambda, E}+\left(1-P_{A, f}(E)\right)\left\langle\sigma_{A}\right\rangle_{A, \bar{E}}$ Lemma 2 shows that we can consider. the conditional average $\langle\sigma\rangle_{A, E}$ instead of 
$\left\langle\sigma_{A}\right\rangle_{\Lambda, f}$. For every configuration $X$ in $E$ each contour in $X$ with endpoints on $\partial \bar{\Lambda}$ splits $\Lambda$ into an "interior" set containing $A$ and an "exterior" set. We call the intersection of all such "interior" sets the set of interior points of $X$, and we call those contours with endpoints on $\partial \bar{\Lambda}$ which form the "boundary" of that set the inner boundary contours. Let $\gamma_{1}, \ldots, \gamma_{n}$ be a family of such contours belonging to some configuration in $E$, and call the corresponding set of interior points $\Lambda^{\prime}$.

Then the conditional average of $\sigma_{A}$ given that the inner boundary contours were precisely $\gamma_{1}, \ldots, \gamma_{n}$ is the average of $\sigma_{A}$ in the box $\Lambda^{\prime}$ with the boundary condition that all spins at the boundary of $\Lambda^{\prime}$ have the same value as indicated in Fig. 2.

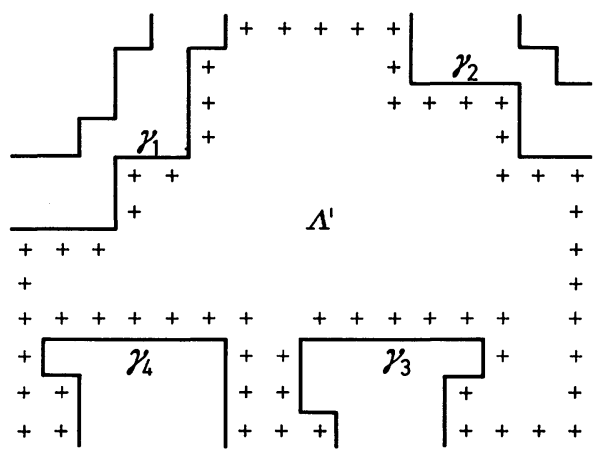

Fig. 2. Boundary condition for given inner boundary contours

(At the corners of the boundary the boundary condition has to be chosen consistent with the rule defined in Fig. 1.) Because neither $\sigma_{A}$ nor the probability of a configuration depends on the value of the boundary spin when $|A|$ is even and $h=0$ the average is not changed if we add the condition that the boundary spin is +1 . This average is however $\geqq\left\langle\sigma_{A}\right\rangle_{A,+}$ $\geqq\left\langle\sigma_{A}\right\rangle_{+}$by the monotonicity of $\left\langle\sigma_{A}\right\rangle_{A,+}$ in $\Lambda$ proved in Lemma 1 . This bound being independent of $\gamma_{1}, \ldots, \gamma_{n}$ we conclude that $\left\langle\sigma_{A}\right\rangle_{A, E} \geqq\left\langle\sigma_{A}\right\rangle_{+}$ also, and the proof is finished by letting $N \rightarrow \infty$.

Remark. That $\left\langle\sigma_{A}\right\rangle_{b}$ is independent of $b$ for high enough temperature is proved in [9], where it is shown that this happens if $m^{*}=0$.

Proof of Lemma 2. Let $\lambda$ be a contour with endpoints on $\partial \bar{\Lambda}$. Then by the argument used in Peierls proof of the existence of a phase transition we can say that the probability that $\lambda$ belongs to a configuration is bounded by: $P_{A, f}(\lambda) \leqq e^{-\beta|\lambda|}$ [5]. Let $\Lambda_{A}$ be a rectangular box with a minimal number of points containing $A$. Then if $\lambda$ separates two points 
of $A$ it must intersect $\bar{\Lambda}_{A}$, so $1-P_{A, f}(E)$ can be bounded as follows:

$$
\begin{aligned}
1-P_{A, f}(E) \leqq & \sum_{p \in \bar{\Lambda}_{A}} \sum_{q, r \in \partial \bar{\Lambda}} \sum_{\lambda \ni p, q, r} e^{-\beta|\lambda|} \leqq \sum_{p \in \bar{\Lambda}_{A}}\left(\sum_{\substack{\lambda \text { ends at } p \\
|\lambda| \geqq N^{1 / 2}}} e^{-\beta|\lambda|}\right)^{2} \\
\leqq & \left|\Lambda_{A}\right|\left(\sum_{l \geqq N^{1 / 2}}\left(3 e^{-\beta}\right)^{l}\right)^{2} \leqq\left|\Lambda_{A}\right|\left(3 e^{-\beta}\right)^{2 N^{1 / 2}}\left(1-3 e^{-\beta}\right)^{-2} \\
& \text { if } 3 e^{-\beta}<1 .
\end{aligned}
$$

It thus goes to zero as $N \rightarrow \infty$ and $P_{\Lambda, f}(E) \rightarrow 1$. (We have used the fact that the number of paths of length $l$ with a given endpoint is at most $3^{l}$.)

To prove the last assertion about $m_{A}$ in Theorem 1 we recall the result of Griffiths [6] that $P_{\Lambda, b}\left(m_{\Lambda} \geqq m^{*}+\varepsilon\right)$ goes to zero for any $\varepsilon>0$ and any $b$. Thus it is enough to show that $\left\langle m_{\Lambda}^{2}\right\rangle_{\Lambda, b}^{1 / 2}$ has a lower bound converging to $m^{*}$. But $\left\langle m_{\Lambda}^{2}\right\rangle_{\Lambda,+} \geqq\left\langle m_{\Lambda}^{2}\right\rangle_{\Lambda, t} \geqq\left\langle m_{\Lambda}^{2}\right\rangle_{\Lambda, f}$ because $\left\langle\sigma_{p} \sigma_{q}\right\rangle_{A,+}$ $\geqq\left\langle\sigma_{p} \sigma_{q}\right\rangle_{\Lambda, t} \geqq\left\langle\sigma_{p} \sigma_{q}\right\rangle_{\Lambda, f}$ by G.K.S., so it is enough to consider $\left\langle m_{\Lambda}^{2}\right\rangle_{\Lambda, f}$. Let $\Lambda^{\prime \prime} \subset \Lambda$ be the box obtained by deleting from $\Lambda$ a "corridor" of width $N^{1 / 2}$ inside $\partial \Lambda$. Then from the proof of Lemma 2 we see that if $A=\{p, q\}$ $1-P_{\Lambda, f}(E) \leqq N \cdot N^{\alpha} \cdot\left(3 e^{-\beta}\right)^{2 N^{1 / 2}}\left(1-3 e^{-\beta}\right)^{-2} \equiv \varepsilon(N)$ when $p, q \in \Lambda^{\prime \prime}$, so it is uniformly small there. We then get (using the fact that $\left\langle\sigma_{p} \sigma_{q}\right\rangle_{\Lambda, f} \geqq 0$ by G.K.S. and the proof of Theorem 2):

$$
\begin{aligned}
\left\langle m_{\Lambda}^{2}\right\rangle_{\Lambda, f}= & \frac{1}{|\Lambda|^{2}} \sum_{p, q \in \Lambda}\left\langle\sigma_{p} \sigma_{q}\right\rangle_{\Lambda, f} \geqq \frac{1}{|\Lambda|^{2}} \sum_{p, q \in \Lambda^{\prime \prime}}\left\langle\sigma_{p} \sigma_{q}\right\rangle_{\Lambda, f} \\
& \geqq \frac{1}{|\Lambda|^{2}} \sum_{p, q \in \Lambda^{\prime \prime}} P_{\Lambda, f}(E)\left\langle\sigma_{p} \sigma_{q}\right\rangle_{\Lambda, E}+\left(1-P_{\Lambda, f}(E)\right)\left\langle\sigma_{p} \sigma_{q}\right\rangle_{\Lambda, \bar{E}} \\
& \geqq \frac{(1-\varepsilon(N))\left|\Lambda^{\prime \prime}\right|^{2}}{|\Lambda|^{2}} \frac{1}{\left|\Lambda^{\prime \prime}\right|^{2}} \sum_{p, q \in \Lambda^{\prime \prime}}\left\langle\sigma_{p} \sigma_{q}\right\rangle_{+}-\varepsilon(N) .
\end{aligned}
$$

This lower bound converges to $\left(m^{*}\right)$ because $\left|\Lambda^{\prime \prime}\right| /|\Lambda| \rightarrow 1$ and

$$
\lim _{|p-q| \rightarrow \infty}\left\langle\sigma_{p} \sigma_{q}\right\rangle_{+}=\left(m^{*}\right)^{2}
$$

by Lemma 1, and the proof is finished.

Remark. It has recently been shown by Gallavotti, Miracle-Solé [2] that any translation invariant set of correlation functions for the infinite system, $\left\langle\sigma_{A}\right\rangle$, is a convex linear combination of $\left\langle\sigma_{A}\right\rangle_{ \pm}$for the same values of $\beta$ as above. This implies that $l_{t}^{*}=m^{*}$ directly, because

$$
\left\langle\sigma_{A}\right\rangle=\lim _{N \rightarrow \infty}\left\langle\sigma_{A}\right\rangle_{\Lambda, t}
$$

is translation invariant. Our result implies that

$$
\left\langle\sigma_{A}\right\rangle_{f}=\left\langle\sigma_{A}\right\rangle_{t}=1 / 2\left(\left\langle\sigma_{A}\right\rangle_{+}+\left\langle\sigma_{A}\right\rangle_{-}\right) .
$$


Acknowledgements. The author has benefitted much from clarifying discussions with G. Gallavotti, R. Griffiths and M. Kac, and has been supported by a travel grant from the Swedish Board for Technical Research.

\section{References}

1. Fisher, M.E.: Phys. Rev. 162, 480 (1967).

2. Gallavotti, G., Miracle-Solé, S.: Equilibrium states of the Ising model in the two phase region. Preprint 1971.

3. Ginibre, J.: Proc. Cargése Summer School 1969. New York: Gordon and Breach 1971.

4. - Commun. math. Phys. 16, 310 (1970).

5. Griffiths, R.: Phys. Rev. A 136, 437 (1964).

6. - Phys. Rev. 152, 240 (1966).

7. - J. Math. Phys. 8, 478, 484 (1967).

8. Kelley, D., Sherman, S.: J. Math. Phys. 9, 466 (1968).

9. Lebowitz,J., Martin-Löf, A.: On the uniqueness of the definition of the critical temperature for Ising ferromagnets. Preprint 1971.

10. Ruelle, D.: On the use of "small external fields" in the problem of symmetry breakdown in statistical mechanics. Preprint 1971.

A. Martin-Löf's present address: Department of Mathematics The Royal Institute of Technology S-10044 Stockholm 70, Sweden 